\title{
Modeling of Combined Deterioration of Concrete Structures by Competing Hazard Model
}

\author{
Kiyoyuki KAITO \\ Associate Professor \\ Frontier Research Center \\ Osaka Univ., Osaka, Japan \\ kaito@ga.eng.osaka-u.ac.jp \\ Kiyoyuki KAITO, born 1972, \\ received his civil engineering \\ degree from the Univ. of Tokyo.
}

\author{
Koichi SUGISAKI \\ Senior Reseacher \\ BMC Corp. \\ Chiba, Japan \\ sugisaki@hashimori.jp
}

Koichi SUGISAKI, born 1976, received civil engineering degree from Tokyo Institute of Technology.

\author{
Kiyoshi KOBAYASHI \\ Professor, Vice Dean \\ Graduate School of \\ Management, Kyoto Univ. \\ Kyoto, Japan \\ kkoba@psa.mbox.media.kyoto- \\ u.ac.jp \\ Kiyoshi KOBAYASHI, born \\ 1952, received civil engineering \\ degree from Kyoto Univ.
}

\section{Summary}

In asset management of infrastructures, predicting deterioration of structures is one of an essential technique. However, in order to estimate their deterioration process with high accuracy, not only single cause deterioration but also combined deterioration appearing on for example concrete structures has to be considered. Therefore this paper addresses the competing hazard model which is possible to model complex deterioration by multiple causes, and especially focuses on that deterioration by neutralization and salt attack on concrete bridges. Specially, separately deteriorations by them are formulated by the Weibull hazard model that can express the timedependent deterioration phenomena. Employing this model, the phenomena of which deterioration probability is increasing with time can be described. Furthermore, the complex deterioration is formulated by neutralization hazard rate's linearly affecting salt attack one.

Keywords: Competing hazard mode; combined deterioration; corrosion; statistical deterioration prediction; asset management.

\section{Introduction}

As a method for maintaining and managing social infrastructures efficiently, asset management is attracting attention. At the first stage of asset management, it is extremely important to predict how deterioration proceeds in structures from a macro perspective. In this circumstance, as deterioration prediction technology, a statistical method, in which a prediction model is statistically developed based on inspection data, has been proposed. Especially, in recent years, a large amount of data have been accumulated through the research into deterioration prediction methods utilizing hazard models[1], which indicates that their practicalities are high. However, the conventional deterioration prediction model targets the damage caused by a single factor and describes its deterioration process. Meanwhile, under the situation where structural and material characteristics are diversified and the usage and environmental conditions vary like social infrastructures, there are many events that have many factors in causing the same damage state. Moreover, such damaging events can be classified into the following two events: (1) deterioration events in which each of several factors proceeds independently and then the most rapid factor becomes significant, and (2) complex deterioration events in which some of several factors are compounded during the deterioration process and accelerate the deterioration process. As a representative complex deterioration, the corrosion of reinforcing bars inside concrete structures can be enumerated. The corrosion of reinforcing bars is considered due to salt damage and neutralization, and there are experimental results that the process of neutralization accelerated the process of salt damage. In addition, the cracking due to the combination of freezing damage $\&$ salt damage or alkali aggregate reaction $\&$ salt damage can be said to be complex deterioration, because if one event proceeds, the other event takes the catalytic role, accelerating the deterioration process. Furthermore, with respect to steel materials, there is no fatigue limit in repeated stress under the corrosion condition, and so there is a possibility that corrosion will increase the probability of fatigue crack no matter the value of repeated stress. Therefore, the development of a model of the deterioration process caused by more than one factor independently or jointly can be said to be a useful technology that would contribute to the advance of asset management. 
Considering the above mentioned issues, the objective of this study is to establish a deterioration prediction model that takes into account compounded deterioration events, by using a competing hazard model. In the following sections of this paper, Chapter 2 formulates a Weibull deterioration hazard model that takes into account the competitive nature among several factors, by using two models that were classified based on whether or not the competitive nature depends on time. Chapter 3 focuses on complex deterioration by the two competing factors: neutralization and salt damage, targeting concrete structures, and estimates the timing of the corrosion of reinforcing bars, using actual inspection data, and empirically analyzes the effectiveness of the proposed model. The following chapters mention the reinforcement corrosion inside concrete structures as a specific target for a competitive deterioration hazard model. Needless to say, it is possible to apply this model to other damage and deterioration phenomena.

\section{Competing Weibull hazard model}

\subsection{Formulation of a competitive hazard model}

The authors construct a model of the complex deterioration phenomenon related to the reinforcement corrosion inside concrete structures, using a hazard model. The target concrete members are represented by $i(i=1, \ldots, K)$, and the elapsed time from the start of use of the member $i$ to present is denoted by $t_{i}$. In addition, it is assumed that there is more than one factor $(j=1, \ldots, J)$ in reinforcement corrosion at the concrete member $i$. Here, it is assumed that the elapsed time $\zeta_{i}$ (lifespan) until reinforcement corrosion occurs at the member is a random variable and is subject to the probability density function $f_{j}\left(\zeta_{i}\right)$ and the distribution function $F_{j}\left(\zeta_{i}\right)$ for each factor $j$. Here, the domain of the life-span is $[0, \infty)$. In addition, the probability $\widetilde{F}_{j}\left(\zeta_{i}\right)$ that reinforcement corrosion will not occur for $t_{i}$ years can be expressed by the following equation:

$\operatorname{Prob}\left\{\zeta_{\mathrm{i}} \geq t_{i}\right\}=\widetilde{F}\left(\zeta_{i}\right)=1-F_{j}\left(\zeta_{i}\right)$

The conditional probability that reinforcement corrosion does not occur at the member $i$ until the arbitrary timing $t_{i}$ and then reinforcement corrosion occurs due to the factor $j$ during the period $\left[t_{i}, t_{i}+\Delta t_{i}\right)$ can be expressed by the following equation:

$\lambda_{j}\left(t_{i}\right) \Delta t_{i}=\frac{f_{j}\left(t_{i}\right) \Delta t_{i}}{\widetilde{F}\left(t_{i}\right)}$

where $\lambda_{j}\left(t_{i}\right)$ represents the hazard function for each corrosion factor $j$, which is discriminated from the hazard function that takes into account all corrosion factors. This equation indicates that the hazard function is probability density. Furthermore, the partial survival distribution function and partial density function for each corrosion factor can be expressed by the following equations, using Equations (1) and (2), respectively [1]:

$$
\begin{aligned}
& \widetilde{F}_{j}\left(t_{i}\right)=\exp \left\{-\int_{0}^{t_{i}} \lambda_{j}(u) d u\right\} \\
& f_{j}\left(t_{i}\right)=\lambda_{j}\left(t_{i}\right) \exp \left\{-\int_{0}^{t_{i}} \lambda_{j}(u) d u\right\}
\end{aligned}
$$

Next, the hazard function for all corrosion factors is defined. Since the survival distribution function for all factors $\widetilde{F}\left(t_{i}\right)$ is the probability that any corrosion factor does not trigger corrosion, that is, the joint probability of the partial survival distribution function for each factor $\widetilde{F}_{j}\left(t_{i}\right)(j=1, \ldots, J)$,

$$
\widetilde{F}\left(t_{i}\right)=\prod_{j=1}^{J} \widetilde{F}_{j}\left(t_{i}\right)
$$

By substituting Equation (3) into Equation (5), the equation can be arranged as follows:

$$
\widetilde{F}\left(t_{i}\right)=\prod_{j=1}^{J} \exp \left\{-\int_{0}^{t_{i}} \lambda_{j}(u) d u\right\}=\exp \left\{-\int_{0}^{t_{i}} \sum_{j=1}^{J} \lambda_{j}(u) d u\right\}
$$

By defining the hazard function for all factors $\lambda\left(t_{i}\right)$ like Equation (3) and comparing it with 
Equation (6), the following equation is obtained.

$$
\lambda\left(t_{i}\right)=\sum_{j=1}^{J} \lambda_{j}\left(t_{i}\right)
$$

Likewise, the probability density function of all factors can be expressed as follows, based on the relation of Equation (2).

$$
f\left(t_{i}\right)=\lambda\left(t_{i}\right) \widetilde{F}\left(t_{i}\right)=\left(\lambda_{1}\left(t_{i}\right)+\cdots+\lambda_{J}\left(t_{i}\right)\right) \widetilde{F}\left(t_{i}\right)=f_{1}\left(t_{i}\right)+\cdots+f_{J}\left(t_{i}\right)=\sum_{j=1}^{J} f_{j}\left(t_{i}\right)
$$

\subsection{Competing Weibull deterioration hazard model}

\subsubsection{Hazard model in which the competitive nature does not depend on time}

A concrete functional form is provided for the hazard function, and the competing hazard model is specified. In this study, the Weibull deterioration hazard model is applied, while assuming that the probability of reinforcement corrosion increases with time. Here, the exponential deterioration hazard model in which the probability of reinforcement corrosion does not depend on time is a special form of the Weibull deterioration hazard model.

Here, as deterioration factors, neutralization (deterioration factor 1), salt damage (deterioration factor 2), and complex deterioration are specified. Suppose that deterioration proceeds inside the concrete structure $i$ because of neutralization (deterioration factor 1) only. The hazard function for deterioration factor 1 at arbitrary timing $t_{i}$ can be defined with the following equation as a general Weibull deterioration hazard model.

$$
\lambda_{1}\left(t_{i}\right)=\gamma_{1} m_{1} t_{i}^{m_{1}-1}
$$

where $\gamma_{1}$ is the parameter representing the arrival rate of corrosion, and $m_{1}$ is the acceleration parameter representing the time dependency of the hazard function. When the acceleration parameter $m_{1}$ is equal to 1 , the hazard function corresponds to the exponential deterioration hazard model that does not depend on time. When the Weibull deterioration hazard function is used, the survival distribution function can be expressed by the following equation:

$$
\widetilde{F}_{1}\left(t_{i}\right)=\exp \left\{-\int_{0}^{t_{i}} \lambda_{1}(u) d u\right\}=\exp \left(-\gamma_{1} t_{i}^{m_{1}}\right)
$$

Next, let us define the hazard function at the timing $t_{i}$ in the case where deterioration proceeds because of salt damage (deterioration factor 2). In this case, the complex deterioration phenomenon between salt damage and neutralization (deterioration factor 1) is also taken into account, and so the following equation is defined:

$$
\lambda_{2}\left(t_{i}\right)=\left\{\gamma_{2}+\delta_{i} \alpha \gamma_{1}\right\} m_{2} t_{i}^{m_{2}-1}
$$

In the above equation, that is, the hazard function for salt damage (deterioration factor 2), it is assumed that when complex deterioration is observed, the arrival rate parameter of the reinforcement corrosion due to deterioration factor $2 \gamma_{2}$ is linearly influenced by the arrival rate parameter due to deterioration factor $1 \gamma_{1}$. Namely, this formulation is made so that the hazard functions for neutralization and salt damage do not influence each other, but neutralization unilaterally influences salt damage. Here, $\delta_{i}$ is the dummy variable:

$$
\delta_{i}= \begin{cases}1 & \text { when complex deterioration occurs } \\ 0 & \text { otherwise }\end{cases}
$$

As mentioned later, it is possible to obtain the information on whether or not complex deterioration has occurred, through visual inspection or the like. In addition, $\alpha$ is the parameter representing the degree of the influence of neutralization on the hazard function for salt damage. When the competitive nature is defined based on Equation (11), the competitive nature becomes constant regardless of time $t$. This basic model is hereinafter called "Model I." In addition, the survival 
distribution function for salt damage (deterioration factor 2) is expressed by the following equation:

$$
\widetilde{F}_{2}\left(t_{i}\right)=\exp \left\{-\int_{0}^{t_{i}} \lambda_{2}(u) d u\right\}=\exp \left\{-\left(\gamma_{2}+\alpha \delta_{i} \gamma_{1}\right) t_{i 2}{ }^{m 2}\right\}
$$

Accordingly, the unknown parameters, which are estimated by the competitive hazard model for reinforcement corrosion, are the following five: the corrosion arrival rate parameters: $\gamma_{1}$ and $\gamma_{2}$, the acceleration parameters: $m_{1}$ and $m_{2}$, and the influence degree parameter: $\alpha$.

\subsubsection{Hazard model in which the competitive nature depends on time}

It is assumed that the speed of the concentration of chloride ion, which is the determinant of reinforcement corrosion, changes (increases) with time. Here, it is possible to define the hazard model for salt damage (deterioration factor 2) taking into account the time dependency of the competitive nature as follows:

$$
\lambda_{2}\left(t_{i}\right)=\left\{\gamma_{2}+\delta_{i} \alpha \lambda_{1}\left(t_{i}\right)\right\} m_{2} t_{i}^{m_{2}-1}
$$

The hazard model in which the time dependency of the competitive nature is described is called Model II. While Model I is constructed under the assumption that the influence of neutralization on salt damage is represented by the corrosion arrival rate parameter $\gamma_{1}$, Model II assumes that this influence is represented by the hazard function $\lambda_{1}\left(t_{i}\right)$. In this case, the survival distribution function for salt damage is expressed by the following equation:

$$
\widetilde{F}_{2}\left(t_{i}\right)=\exp \left\{-\int_{0}^{t_{i}} \lambda_{2}(u) d u\right\}=\exp \left\{-\int_{0}^{t_{i}}\left\{\gamma_{2}+\alpha \delta_{i} \lambda_{1}(u)\right\} m_{2} u^{m_{2}-1} d u\right\}=\exp \left\{-\gamma_{2} t_{i}^{m_{2}}-\alpha_{1} \delta_{i} \gamma_{1} m_{1} m_{2} \frac{t_{i}^{m_{1}+m_{2}-1}}{m_{1}+m_{2}-1}\right\}
$$

\subsection{Estimation method}

\subsubsection{Contents of inspection data}

Suppose that $K$ visual inspection data regarding concrete structures have been obtained. The inspection data is composed of mainly "inspection results" and "characteristic information." Inspection results have the information on whether or not reinforcement corrosion has occurred and the factors in corrosion (neutralization, salt damage and complex deterioration) for each inspection data sample $i(i=1, \ldots, K)$. On the other hand, for characteristic information, the structural, usage, and environmental conditions that would influence reinforcement corrosion are recorded.

Firstly, inspection results are described. Out of $K$ inspection data samples, the samples in which reinforcement corrosion has occurred are classified as follows, for each factor: neutralization and salt damage. (Since complex deterioration is expressed in the hazard function for salt damage, this is included in the salt damage samples in this discussion.) The population of the samples in which reinforcement corrosion has occurred due to the factor $j$ is represented by $\Phi^{j}$, and the total number of such samples is represented by $K(j)$, and the $k$-th sample of $\Phi^{j}$ is denoted by $(j, k)$. In the above discussions, samples are distinguished by using " $i$ " but in the following discussions, samples are discriminated by using $(j, k)$. Accordingly, the population of the samples in which reinforcement corrosion has occurred due to neutralization (deterioration factor $1, j=1$ ) is represented by $\Phi^{1}$, the total number of such samples is $K(1)$; those for salt damage (deterioration factor $2, j=2$ ) are $\Phi^{2}$ and $K(2)$, respectively. On the other hand, the sound condition samples in which reinforcing bars did not corrode are defined as $j=0$. Obviously, there is the following relation:

$$
K(0)+K(1)+K(2)=K
$$

In this study, it is assumed that after the occurrence of reinforcement corrosion is confirmed, corrosion factors are identified through inspection. In this assumption, ordinary visual inspection is considered. However, it is noteworthy that corrosion factors can be identified before the occurrence of reinforcement corrosion through the core-sampling test or the like. Based on the above mentioned definitions and assumptions, it is possible to describe the information on the occurrence of reinforcement corrosion and the factors in corrosion (neutralization and salt damage), regarding 
concrete structures $(j, k)$. In addition, the elapsed time of the sample $(j, k)$ is represented by $t_{j k}$. With regard to complex deterioration, the dummy variable in Equation (12) is redefined as $\delta_{j k}$, and in the salt damage sample $(2, k)$, it is possible to check whether or not complex deterioration is the corrosion factor.

Next, characteristic information is described. It is assumed that the start timing of the corrosion of reinforcing bars depends on the material and environmental characteristics of concrete structures. Accordingly, the structural, usage, and environmental characteristics that would influence the corrosion factors: neutralization and salt damage are specified. For each factor, the characteristic vector is represented by $\mathbf{x}^{j k}=\left(\mathbf{x}_{1}^{j k}, \mathbf{x}_{2}^{j k}\right)$. Here, the subscripts 1 and 2 denote neutralization and salt damage, respectively. In addition, $\mathbf{x}_{1}^{j k^{2}}=\left(x_{1}^{j k}, \ldots ., x_{e 1}^{j k}\right), \mathbf{x}_{2}^{j k}=\left(x_{1}^{j k}, \ldots ., x_{e 2}^{j k}\right)$, and $e 1$ and $e 2$ represent the total numbers of characteristic information that would influence respective factors. Accordingly, the information on concrete structures $(j, k)$ that can be obtained through visual inspection can be expressed by $\xi^{j k}=\left(t_{j k}, \delta_{j k}, \mathbf{x}^{j k}\right)$.

\subsubsection{Estimation of unknown parameters with the maximum-likelihood method}

In order to estimate the competing Weibull deterioration hazard model based on the inspection data population $\xi$, it is assumed that the differences in corrosion occurrence due to the structural, usage, and environmental conditions can be expressed by the arrival rate of corrosion: $\gamma_{j k}$, which is represented by a characteristic vector as follows:

$\gamma_{j k}=\exp \left(\boldsymbol{\beta}_{j} \mathbf{x}^{j k}{ }^{\prime}\right) \quad j=1,2$

where $\boldsymbol{\beta}_{j}=\left(\beta_{j}{ }^{1}, \beta_{j}{ }^{2}, \cdots, \beta_{j}{ }^{e j}\right)$ is the row vector composed of unknown parameters. The symbol ' in the above equation denotes transposition. Accordingly, the characteristic information on each concrete structure: $\mathbf{x}^{j k}$, which can be obtained through inspection, is reflected in the arrival rate parameter. Therefore, the later-mentioned estimation of unknown parameters can be considered as the estimation of $\boldsymbol{\beta}_{j}$ rather than the direct estimation of the arrival rate parameter $\gamma_{j k}$. Then, the unknown parameters that should be estimated are $\boldsymbol{\beta}_{1}, \boldsymbol{\beta}_{2}, m_{1}, m_{2}$, and $\alpha$. These are represented by $\boldsymbol{\theta}=(\boldsymbol{\beta}, \mathbf{m}, \alpha)$.

Next, when the $k$-th sample $(j, k)$ in which corrosion factor $j(j=1,2)$ induces reinforcement corrosion is focused on, the likelihood function of the sample can be expressed by the following equation, based on Equations (2) and (5).

$$
L_{j k}(\theta)=f_{j}\left(t_{j k}\right)=\lambda_{j}\left(t_{j k}\right) \widetilde{F}\left(t_{j k}\right)=\lambda_{j}\left(t_{j k}\right) \widetilde{F}_{1}\left(t_{j k}\right) \cdots \widetilde{F}_{j}\left(t_{j k}\right)=\lambda_{j}\left(t_{j k}\right) \prod_{h=1}^{J} \widetilde{F}_{h}\left(t_{j k}\right)
$$

On the other hand, the likelihood function of the sound condition sample (either factor does not induce damage; $j=0$ ), in which reinforcement corrosion did not occur, is expressed as follows:

$$
L_{0 k}(\theta)=\widetilde{F}\left(t_{0 k}\right)=\prod_{h=1}^{J} \widetilde{F}_{h}\left(t_{0 k}\right)
$$

Furthermore, the sum of log likelihoods for all samples is expressed as follows:

$$
\ln L(\theta)=\sum_{j=0}^{J} \sum_{k=1}^{K(j)} \ln L_{j k}(\theta)
$$

When the maximum-likelihood method is used, the maximum likelihood estimator of the parameter $\theta$ that maximizes the $\log$ likelihood function (20) can be obtained as $\hat{\theta}$ that satisfies the following relation:

$$
\frac{\partial \ln L(\hat{\theta})}{\partial \theta}=0
$$


Table 1 Overview of Used Data (total number of samples: 145)

\begin{tabular}{c|c|c}
\hline & Average & Standard Deviation \\
\hline Age & 46.2 & 19.4 \\
Neutralization Depth [mm] & 38.3 & 9.0 \\
Chloride Ion Concentration at Reinforcing Bars $\left[\mathrm{kg} / \mathrm{m}^{3}\right]$ & 1.1 & 1.4 \\
\hline
\end{tabular}

Table 2 Reference Values for Estimating Reinforcement Corrosion

\begin{tabular}{c|c|c}
\hline & Used data & Reference Value \\
\hline Neutralization & $\begin{array}{c}\text { Neutralization residue } \\
\text { Chloride ion concentration } \\
\text { (whole salt content) }\end{array}$ & $25 \mathrm{~mm}$ \\
Complex deterioration & Initial chloride ion amount & $1 \mathrm{~kg} / \mathrm{m}^{3}$ \\
$1 \mathrm{~kg} / \mathrm{m}^{3}$ \\
\hline
\end{tabular}

\section{Empirical Study}

\subsection{Overview of inspection data}

In this study, the authors could use the diagnostic data of the core extraction test, which is detailed investigation, in addition to visual inspection. Table 1 shows the averages and variances of age, neutralization depth, and chloride ion concentration at reinforcing bars, which are considered to influence reinforcement corrosion, for all of 145 samples. The samples were taken from concrete structures near the shoreline, and so chloride ion concentration is high as a whole. With regard to age, variation is relatively large. The necessary explained variables for this model are the two values representing whether or not reinforcement corrosion has occurred. However, in order to estimate the competing deterioration hazard model, it is necessary to clarify which is the factor in reinforcement corrosion; neutralization, salt damage, or complex deterioration. In this study, in order to infer the factor in reinforcement corrosion, the authors focused on "neutralization residue" for neutralization, "chloride ion concentration" for salt damage, and "initial chloride ion amount" for complex deterioration, and then obtained actual measurement values through the core extraction method targeted at corrosion-seen samples. The factor in reinforcement corrosion was identified by comparing the actual measurement values with the reference values (thresholds) shown in Table 2 . Practically, there are some cases in which it is impossible to conduct the core extraction test for all samples. In such a case, the factor in reinforcement corrosion is determined based on the subjective judgment by inspectors or engineers.

With regard to neutralization, the reference value of neutralization residue in the specification is 10 $\mathrm{mm}$, but it is known that the actual measurement value of covering depth varies considerably. Therefore, with regard to neutralization, considering safety and assuming that the specified value of covering depth varies from $15 \mathrm{~mm}$ to $40 \mathrm{~mm}$, it was recognized that when neutralization residue exceeds $25 \mathrm{~mm}$, reinforcement corrosion occurs. With regard to salt damage, it was recognized that reinforcement corrosion occurs when the salt content at reinforcing bars exceeds $1 \mathrm{~kg} / \mathrm{m}^{3}$; this value was specified considering that the samples were taken in the vicinity of the shoreline and emphasizing safety while the reference value in the specification is $1.2 \mathrm{~kg} / \mathrm{m}^{3}$. With regard to complex deterioration, the authors judged by checking whether or not initial chloride ion concentration is above the reference value by referring to the previous study. With regard to complex deterioration, it is acceptable to judge by seeing the distribution form of chloride ion concentration, but it has been pointed out that complex deterioration is highly correlated with initial chloride ion concentration, and so the authors decided to adopt the former one in this study. In some cases, there were samples in which the values of neutralization and salt damage exceed the respective reference values. Such samples were classified into complex deterioration. 
Table 3 Estimated Parameters

\begin{tabular}{c|c|c|c|c|c|c}
\hline & $\beta_{1}$ & $m_{1}$ & $\beta_{2}{ }^{1}$ & $\beta_{2}{ }^{2}$ & $m_{2}$ & $\alpha$ \\
\hline Model I & -16.14 & 3.30 & -13.48 & 2.02 & 2.93 & 63.49 \\
Model II & -33.55 & 7.36 & -7.84 & 2.27 & 1.60 & 0.45 \\
\hline
\end{tabular}

\subsection{Estimation of competing hazard model}

Utilizing inspection data, the authors conduct the estimation of the competing Weibull deterioration hazard model, which was formulated in Section 2. From the information that can be obtained through inspection, the explanatory variables of the hazard models for neutralization and salt damage were selected through trial and error. As a result, there were no data that can be used as explanatory variables for neutralization, and the surface chloride ion amount was adopted for salt damage. Accordingly, the concrete hazard functions for neutralization $\gamma_{1}$ and salt damage $\gamma_{2 k}$ can be defined as follows:

$$
\gamma_{1}=\exp \left(\beta_{1}{ }^{1}\right), \quad \gamma_{2 k}=\exp \left(\beta_{2}{ }^{1}+\beta_{2}{ }^{2} x_{2}^{2 k}\right)
$$

where $x_{2}^{j k}$ is the surface chloride ion concentration of the sample $(j, k)$ that was normalized so that its maximum is equal to 1 . The neutralization hazard function: $\gamma_{1}$ is constant regardless of the sample $(j, k)$, because the explanatory variable is composed of a constant term only. It is noteworthy that $\gamma_{1}$ does not have the subscript $k$. In addition, as mentioned in Section 2.2.1, when the competitive deterioration hazard model in which the competitive nature does not depend on time: Model I is adopted, the log likelihood for all samples becomes as follows:

$$
\begin{aligned}
\ln L(\theta & : \xi)=\sum_{j=0}^{2} \sum_{k=1}^{K(j)} \ln L_{j k}=\sum_{k=1}^{K(0)} \ln L_{0 k}+\sum_{k=1}^{K(1)} \ln L_{1 k}+\sum_{k=1}^{K(2)} \ln L_{2 k} \\
= & \sum_{k=1}^{K(0)}\left\{-\gamma_{1} t_{0 k}{ }^{m_{1}}-\left(\gamma_{2 k}+\alpha \delta_{0 k} \gamma_{1}\right) t_{0 k}{ }^{m_{2}}\right\}+\sum_{k=1}^{D(1)}\left\{\ln \left[\gamma_{1} m_{1} t_{1 k}{ }^{{ }_{1}-1}\right]-\gamma_{1} t_{1 k}{ }^{m_{1}}-\left(\gamma_{2 k}+\alpha \delta_{1 k} \gamma_{1}\right) t_{1 k}{ }^{{ }_{2}}\right\} \\
& +\sum_{k=1}^{D(2)}\left\{\ln \left[\left\{\gamma_{2 k}+\alpha \delta_{2 k} \gamma_{1}\right\} m_{2} t_{2 k}{ }^{m_{2}-1}\right]-\gamma_{1} t_{2 k}{ }^{m_{1}}-\left(\gamma_{2 k}+\alpha \delta_{2 k} \gamma_{1}\right) t_{2 k}{ }^{{ }^{2}}\right\}
\end{aligned}
$$

On the other hand, when the competing deterioration hazard model in which the competitive nature depends on time: Model II is used, the log likelihood for all samples can be formulated as follows:

$$
\begin{aligned}
& \ln L(\theta: \xi) \\
& =\sum_{k=1}^{K(0)}\left\{-\gamma_{1} t_{0 k}^{m_{1}}-\gamma_{2 k} t_{0 k}^{m_{2}}-\alpha \delta_{0 k} \gamma_{1} m_{1} m_{2} \frac{t_{0 k}^{{ }^{m_{1}+m_{2}-1}}}{m_{1}+m_{2}-1}\right\}+\sum_{k=1}^{K(1)}\left\{\ln \left[\gamma_{1} m_{1} t_{1 k}{ }^{m_{1}-1}\right]-\gamma_{1} t_{1 k}{ }^{m_{1}}-\left(\gamma_{2 k}+\alpha \delta_{1 k} \gamma_{1}\right) t_{1 k}{ }^{m_{2}}\right\} \\
& +\sum_{k=1}^{K(2)}\left[\ln \left\{\left(\gamma_{2 k}+\alpha \delta_{2 k} \gamma_{1} m_{1} t_{2 k}{ }^{m_{1}-1}\right) m_{2} t_{2 k}{ }^{m_{2}-1}\right\}-\gamma_{1} t_{2 k}{ }^{m_{1}}-\gamma_{2 k} t_{2 k}{ }^{m_{2}}-\alpha \delta_{2 k} \gamma_{1} m_{1} m_{2} \frac{t_{2 k}{ }^{m_{1}+m_{2}-1}}{m_{1}+m_{2}-1}\right]
\end{aligned}
$$

The unknown parameters that maximize the above equations (24) and (25) are estimated with the direct search method. The estimation results are shown in Table 3. Both of the acceleration parameters are above 1. This indicates the characteristics of the Weibull deterioration hazard type rather than the exponential deterioration hazard type. Especially, it is obvious that the influence of the acceleration parameter for neutralization $m_{1}$ becomes stronger with time. In addition, $\alpha$ in Model I is larger than that in Model II. It can be considered that the time dependency of the competitive nature is taken into account in Model II, and so the complex deterioration process is described even if $\alpha$ is small.

\subsection{Analytical results}

With the hazard model, it is possible to formulate survival probability. If the probability that 


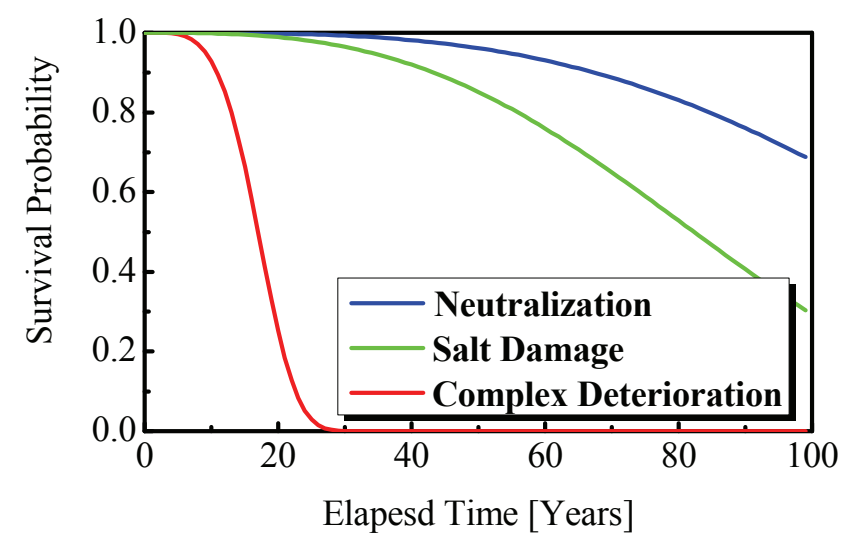

Fig. 1 Survival Probability: Model I

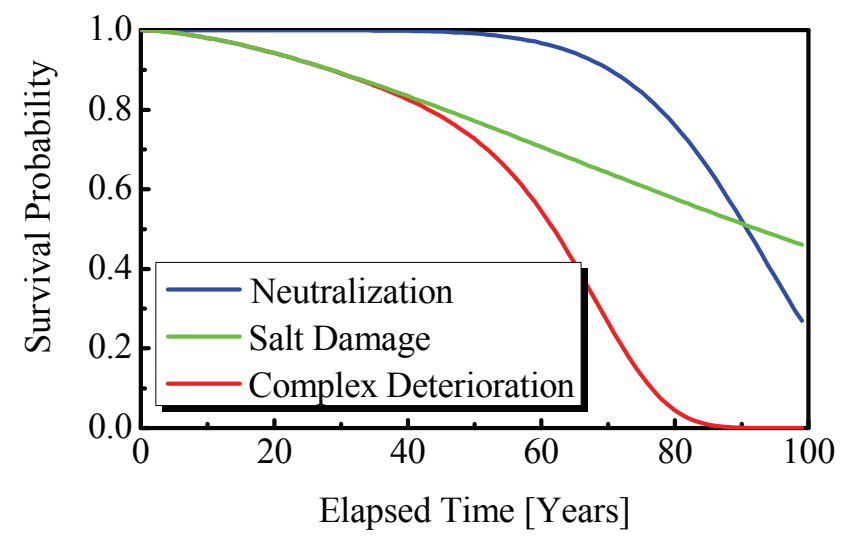

Fig.2 Survival Probability: Model II

reinforcement corrosion occurs due to the corrosion factor $j$ at arbitrary timing $\tau_{i}$ is called survival probability, it can be calculated by solving the distribution function for each factor. Figs. 1 shows the survival probabilities in Model I, which was calculated using the estimation results mentioned in the previous section. Fig. 1 shows the survival curve calculated using the average of surface chloride ion concentration. For the figure, the high factors in reinforcement corrosion are complex deterioration, salt damage, and neutralization in this order. Therefore, it can be concluded that the complex deterioration proceeds more rapidly than the deterioration caused by a single factor of neutralization or salt damage. In detail, the period until the survival probability against reinforcement corrosion due to complex deterioration, salt damage, and neutralization reaches 0.5 is about 17 years, about 83 years, and about 130 years, respectively in Fig. 1. It is noteworthy that the reason why neutralization is slow is that the samples used for this empirical analysis were taken from concrete structures near the shoreline and so there were few samples of neutralization. Actually, the number of samples of neutralization was only 4 out of a total of 145 samples.

On the other hand, Figs. 2 shows Model II in which the competitive nature depends on time. With regard to the survival curves calculated from the average of surface chloride ion concentration, the complex deterioration proceeds like the deterioration due to salt damage at the early stage, but the probability of corrosion steeply increases from a certain age. In addition, as obvious from the results in Table 3, in the present model that takes into account the time dependency of the competitive nature, the corrosion due to neutralization occurs earlier and its influence on complex deterioration is larger. These are the reason why the corrosion due to neutralization is earlier and the corrosion due to salt damage is slower in this figure than Fig. 1.

\section{Conclusions}

In this study, the authors proposed a competing hazard model taking into account the competitive nature among several factors, targeting the complex deterioration phenomenon at infrastructures. Focusing on the estimation of the timing of the start of reinforcement corrosion inside concrete, the authors confirmed that the competitive nature between neutralization and salt damage accelerates the start of reinforcement corrosion and studied when the effect of complex deterioration becomes significant, through the empirical analysis using actual inspection data. Accordingly, it is expected that the proposed model will enable the description of the complex deterioration phenomenon at social infrastructures and contribute to the advance of asset management.

Part of this study was conducted at Frontier Research Base for Global Young Researchers, Graduate School of Engineering, Osaka University, supported by Special Coordination Funds for Promoting Science and Technology, Ministry of Education, Culture, Sports, Science and Technology.

\section{Reference}

[1]TSUDA, Y., KAITO, K., AOKI, K. and KOBAYASHI, K.: Estimating Markovian Transition Probabilities for Bridge Deterioration Forecasting, Journal of Structural Eng./Earthquake Eng., JSCE, Vol.23, No.2, pp.241s-256s, 2006. 